\title{
A Proposal for the Diagnosis and Treatment of Hypersensitivity State in Migraine
}

\author{
Toshihiko Shimizu ${ }^{1}$, Koichi Hirata ${ }^{2}$, Shinya Manaka ${ }^{3}$, Yoshikazu Okada ${ }^{1}$ and Ichiro Arakawa ${ }^{4}$ \\ 1. Department of Neurosurgery, Institute of Neurology, Tokyo Women's Medical University, Shinjuku City 162-0054, Tokyo, Japan \\ 2. Department of Neurology, Dokkyo Medical University, Mibu 321-0293, Tochigi, Japan \\ 3. Manaka Hospital, Odawara City 250-0012, Kanagwa, Japan \\ 4. Faculty of Pharmaceutical Science, Teikyo Heisei University, Nakano City 164-8530, Tokyo, Japan
}

\begin{abstract}
The pathophysiology of migraines has been commonly explained by the trigeminovascular theory, although recent studies have suggested that the cause of migraines stems from cortical hyperexcitability. The aim of this study was to demonstrate the effectiveness of antiepileptic drugs for treating patients with cephalic hypersensitivity symptoms in Japan. In this study, we assessed the (1) demographics; (2) medical history; (3) treatment status and (4) electroencephalograms of the subjects. This study was performed after approval by the ethics committee at Tokyo Women's Medical University. We examined the electroencephalograms of 1,616 patients experiencing daily headaches accompanied by dizziness and cephalic ringing. Of these patients, approximately $80 \%$ had migraine without aura, and were asymptomatic before migraine onset. In addition, we measured the effects, dosages, and treatment durations of anti-epileptic drugs commonly prescribed as migraine prophylactics in 172 patients (age \pm standard deviation: $55.8 \pm 14.3$ years) with cephalic hypersensitivity symptoms. Our results suggested that the electroencephalogram abnormalities related to cephalic hypersensitivity symptoms were associated with age and photophobia. Anti-epileptic drugs for cephalic hypersensitivity symptoms may be effective for reducing cerebral hypersensitivity. Statistics show that the drugs were markedly effective in about $28 \%$ of patients, moderately effective in $52 \%$ of patients, and not effective in $20 \%$ of patients. Our study suggests that erroneous migraine treatment methods from childhood may exacerbate hypersensitivity of the brain leading to the development of dizziness, tinnitus, or cephalic ringing, and shows that anti-epileptics are effective for treating this condition.
\end{abstract}

Key words: Hypersensitivity, migraine, prophylactic medication, EEG.

\section{Introduction}

It has recently been argued that patients who tend to experience migraines also exhibit hypersensitivity of the brain from childhood [1]. In particular, at migraine onset, an excitation wave originating in the medial occipital lobe, which is located in the visual cortex, is propagated toward the cerebral frontal lobe, with the excitatory symptoms in the occipital lobe appearing as an aura known as a central scotoma. The major pharmacological mechanisms of triptans are believed to act on serotonin (5-HT, 5-hydroxytryptamine;) 1B receptors, found in the walls of cerebral blood vessels,

Corresponding author: Toshihiko Shimizu, M.D., Ph.D., guest professor, research field: neurosurgery (headache). E-mail: headache.shimizu@k4.dion.ne.jp. and on $5-\mathrm{HT}_{1 \mathrm{D}}$ receptors, found in trigeminal nerve endings surrounding the cerebral vessels. Furthermore, some medications, albeit only a few, also act on $5-\mathrm{HT}_{1 \mathrm{~F}}$ receptors in the trigeminal nucleus. These drugs were bound to the receptors during the time that migraine attacks to inhibit neurotransmission within the trigeminal nucleus, thereby fundamentally aborting the migraine attacks [2].

Misuse of NSAIDs (non-steroidal anti-inflammatory drugs) or enduring attacks while bypassing the appropriate treatment results in the continuation of a state of sensitized central hypersensitivity; however, as irritation of the trigeminal nerve around blood vessels declines, complaints of headache itself diminish or disappear. Nevertheless, the brain remains in a sensitized state, 
which has its roots in the trigeminal nucleus of the brainstem. Alternatively, although the pain declines because the pain pathway from the thalamus to the prefrontal area of the cerebral cortex loses its excitability over time, the thalamus remains in a sensitized state. The sensitized state of the thalamus is propagated to the contralateral cerebral hemisphere via the thalamocerebellar tract, causing dizziness and tinnitus in patients with migraine who have failed to undergo appropriate treatment [3]. Furthermore, the hypersensitivity to sound that occurs during migraines may develop into cephalic ringing, caused by severe chronic hyperexcitation of the temporal lobe [4]. Ueyamal et al. [5] also reported a magnetic resonance imaging study that suggested dizziness may be caused by hyperexcitation of certain areas of the brain.

We have previously reported that such erroneous migraine treatment methods from childhood may exacerbate the hyperexcitability of the brain and cause dizziness, tinnitus, or cephalic ringing to develop [6]. In a recent study, Han et al. [7] carried out a randomized crossover trial of drugs that act on gamma-aminobutyric acid receptors for cephalic ringing, and found that clonazepam was an effective treatment for cephalic ringing. Similar to the report by Han et al. [7], we carried out a retrospective study to demonstrate the effectiveness of anti-epileptic drugs for treating patients with CHS (cephalic hypersensitivity symptoms) in Japan, the results of which are reported herein.

\section{Methods}

\subsection{Participants}

A total of 1,616 patients who had been examined at participating medical institutions and met the following selection criteria were isolated from clinical records databases: (1) complaint of dizziness or cephalic ringing; (2) migraine with or without aura based on the International Classification of Headache Disorders, 2nd edition (ICHD-II) International Diagnostic Criteria for Migraine [8] and (3) EEG (electroencephalogram) results available.

\subsection{Study Design}

This was a retrospective single cohort study designed to evaluate the burden of disease in patients with CHS and to determine the efficacy of treatment.

\subsection{Observations}

Date of birth, sex, and complications (depression, dizziness, etc.), were recorded from clinical records as the patients' background information. In addition, the EEG test results (Figs. 1a and $1 \mathrm{~b}$ show typical abnormal and normal EEGs, respectively) were documented as clinical findings, and the use of triptan medications and anti-epileptics (valproate sodium, clonazepam, topiramate, etc.) were noted.

\subsection{Statistical Analysis}

The following demographic characteristics were calculated: mean age $\pm \mathrm{SD}$, sex distribution, mean duration of disease $\pm \mathrm{SD}$, and percentages of complications. Logistic regression analysis was performed, with the demographic characteristic indices, EEG findings, and clinical findings as independent variables in order to explore factors contributing to the EEG abnormalities. A sub-analysis of patients who used anti-epileptics was also performed to evaluate the therapeutic efficacy of the drugs, and the frequency distributions of their efficacies (marked response, moderate response, or no change) were calculated. Survival analysis was also performed using the Kaplan-Meier method to explore the correlation between the therapeutic efficacy of anti-epileptics and their duration of use.

\subsection{Ethical Considerations}

This study was performed after approval by the ethics committee at Tokyo Women's Medical University in compliance with the ethical guidelines for epidemiological and clinical studies published by the Ministry of Health, Labour, and Welfare and the 


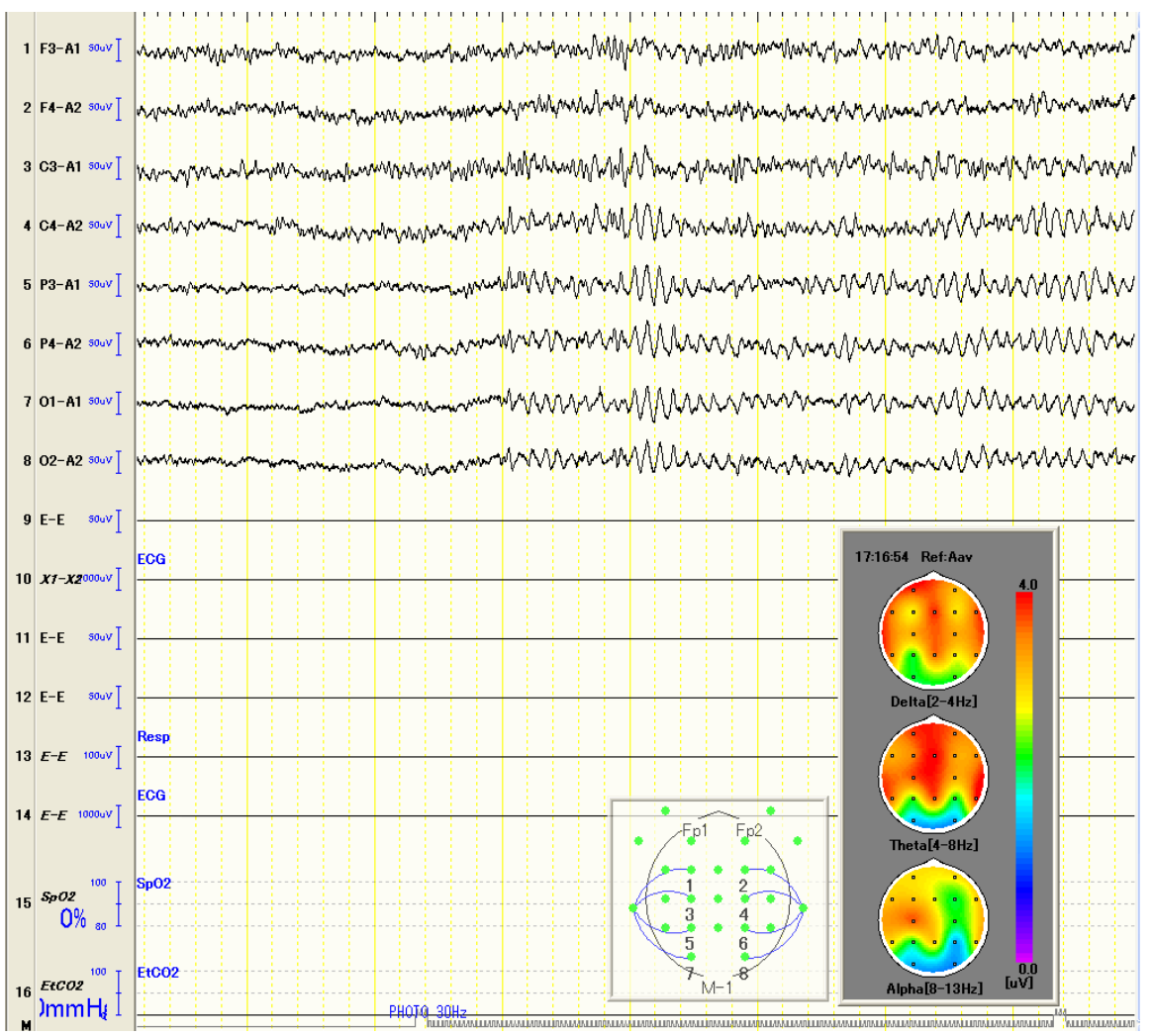

(a)

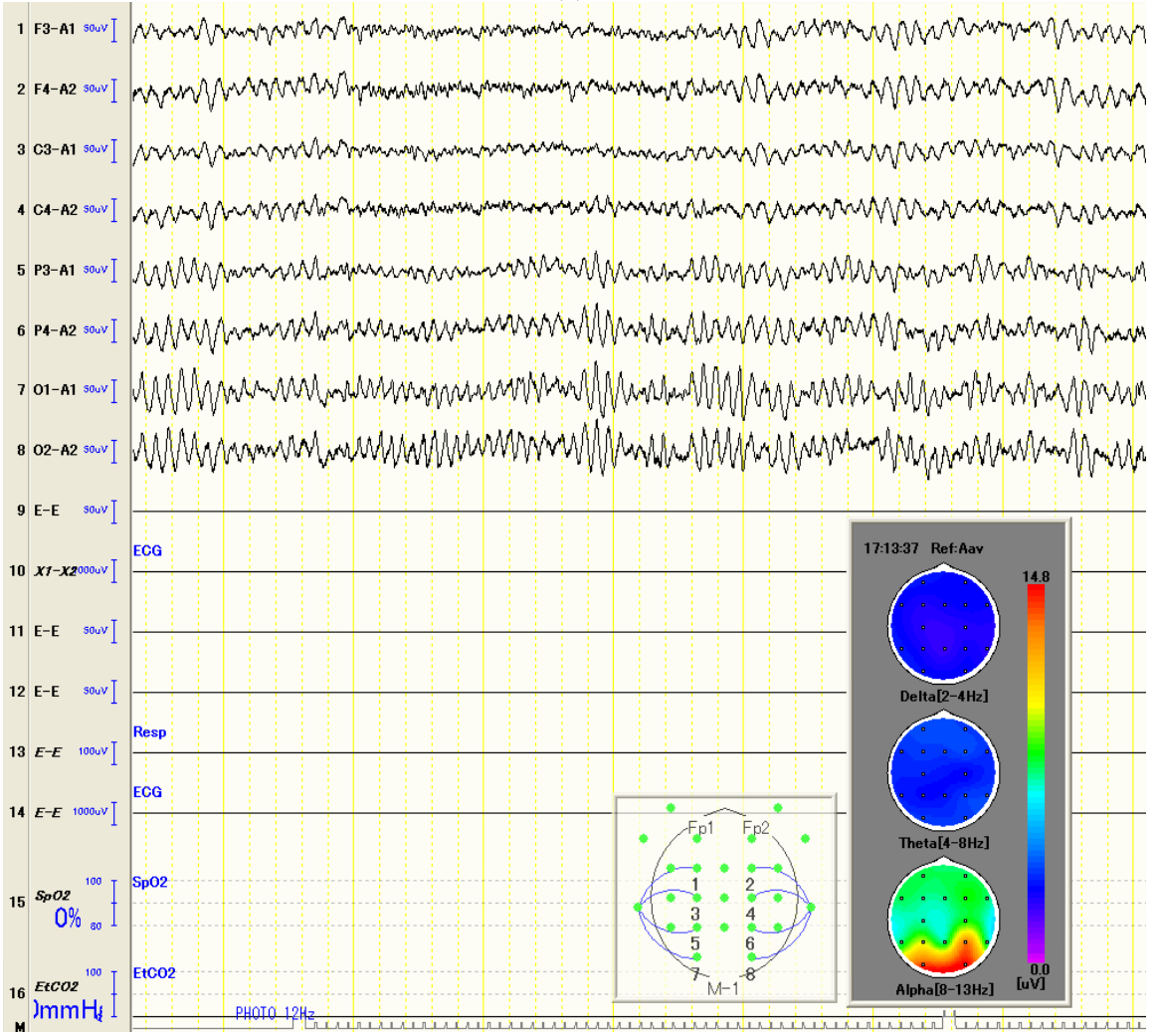

(b)

Fig. 1 Electroencephalogram of a patient diagnosed CHS (a) abnormal and (b) normal. 
Ministry of Education, Culture, Sports, Science, and Technology.

\section{Results}

\subsection{Demographic Characteristics}

The mean age of the 1,616 patients was $45.68 \pm$ 15.2 years, with women accounting for approximately $77 \%$ of patients.

\subsection{Characteristics of the Clinical Findings}

Approximately $4 \%$ of patients also experienced depression, and approximately $15 \%$ of patients experienced dizziness.

\subsection{Exploration of Factors Affecting EEG} Abnormalities

Results showed that age and temporal proximity to photostimulation were significant factors affecting the EEG abnormalities (Table 1).

\subsection{Effectiveness of Anti-epileptics}

Of the 1,616 patients, the effectiveness of anti-epileptics could be evaluated in 172 patients. The calculated frequency distributions showed that the drugs had a marked response in $28 \%$ of patients and a moderate response in $52 \%$ of patients, thus displaying therapeutic efficacy in $80 \%$ of patients (Fig. 2). As the duration of anti-epileptic use differed between patients, survival analysis was also performed using the Kaplan-Meier method. The results of this analysis showed that the median time to improvement was 294.0 days (95\% confidence interval: [237.0, 351.0]) (Fig. 3).

\section{Discussion}

We have previously reported that erroneous migraine treatment methods from childhood may exacerbate hyperexcitability of the brain and cause dizziness, tinnitus, or cephalic ringing to develop [4]. In this study, we increased the number of patients, explored factors contributing to EEG abnormalities in patients showing therapeutic medication effects, and investigated the efficacy of anti-epileptics. We found that age and temporal proximity to photostimulation were significant factors associated with EEG abnormalities. A therapeutic effect of anti-epileptics was evident in $80 \%$ of patients, and the median time to improvement was 294.0 days. These results establish that the use of anti-epileptics is effective for treating CHS.

Table 1 Logistic regression model of electroencephalogram abnormality to explore related factors.

\begin{tabular}{|c|c|c|c|c|c|}
\hline \multirow{2}{*}{ Waning } & & \multirow{2}{*}{$P$ value } & \multirow{2}{*}{ OR } & \multicolumn{2}{|c|}{$95 \% \mathrm{CI}$ of OR } \\
\hline & & & & Lower & Upper \\
\hline \multirow{14}{*}{ Absent } & Coefficient & 0.330 & & & \\
\hline & Gender & 0.402 & 0.880 & 0.652 & 1.187 \\
\hline & Age & 0.043 & 1.009 & 1.000 & 1.017 \\
\hline & Spike & 0.983 & 0.987 & 0.305 & 3.192 \\
\hline & Photo (pre) -ve & 0.000 & 3.745 & 2.748 & 5.104 \\
\hline & Photo (pre) +ve & & & & \\
\hline & Migraine -ve & 0.723 & 0.925 & 0.603 & 1.421 \\
\hline & Migraine +ve & & & & \\
\hline & Sudden deafness -ve & 0.315 & 1.656 & 0.619 & 4.432 \\
\hline & Sudden deafness +ve & & & & . \\
\hline & Tinnutus -ve & 0.537 & 1.370 & 0.504 & 3.722 \\
\hline & Tinnutus +ve & & & & \\
\hline & Photo (post) -ve & 0.001 & 1.770 & 1.250 & 2.504 \\
\hline & Photo (post) +ve & & & & \\
\hline
\end{tabular}

$n=1,616$; OR: odds ratio; CI: confidence interval; +ve: positive; -ve: negative. 


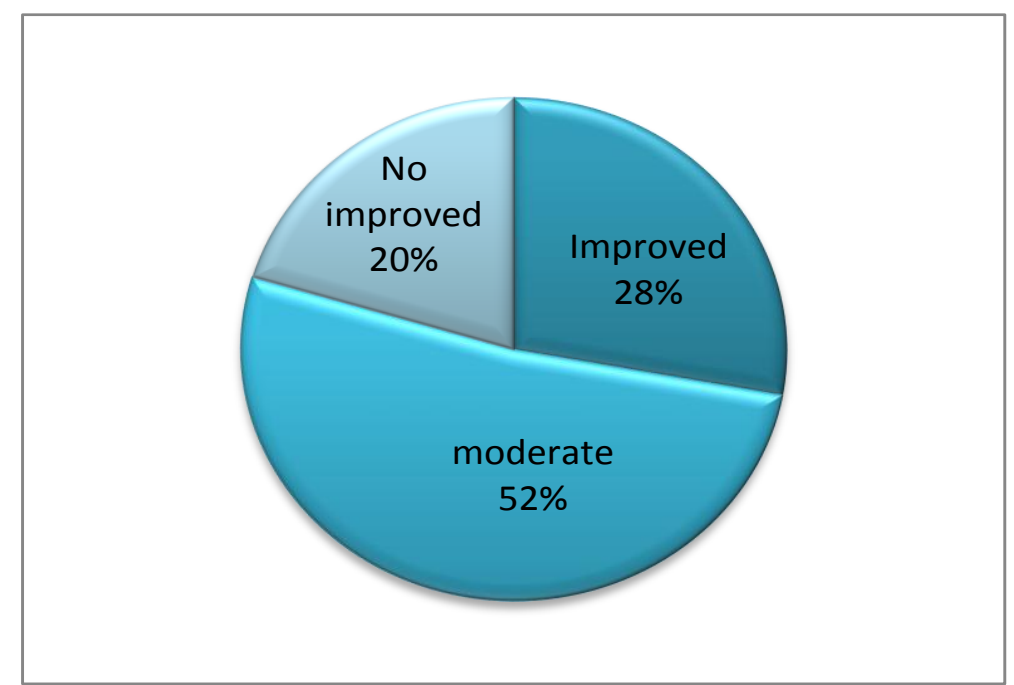

Fig. 2 Effect of Anti-epileptic drugs (Sodium Valproate and Clonazepam) for the treatment of CHS.

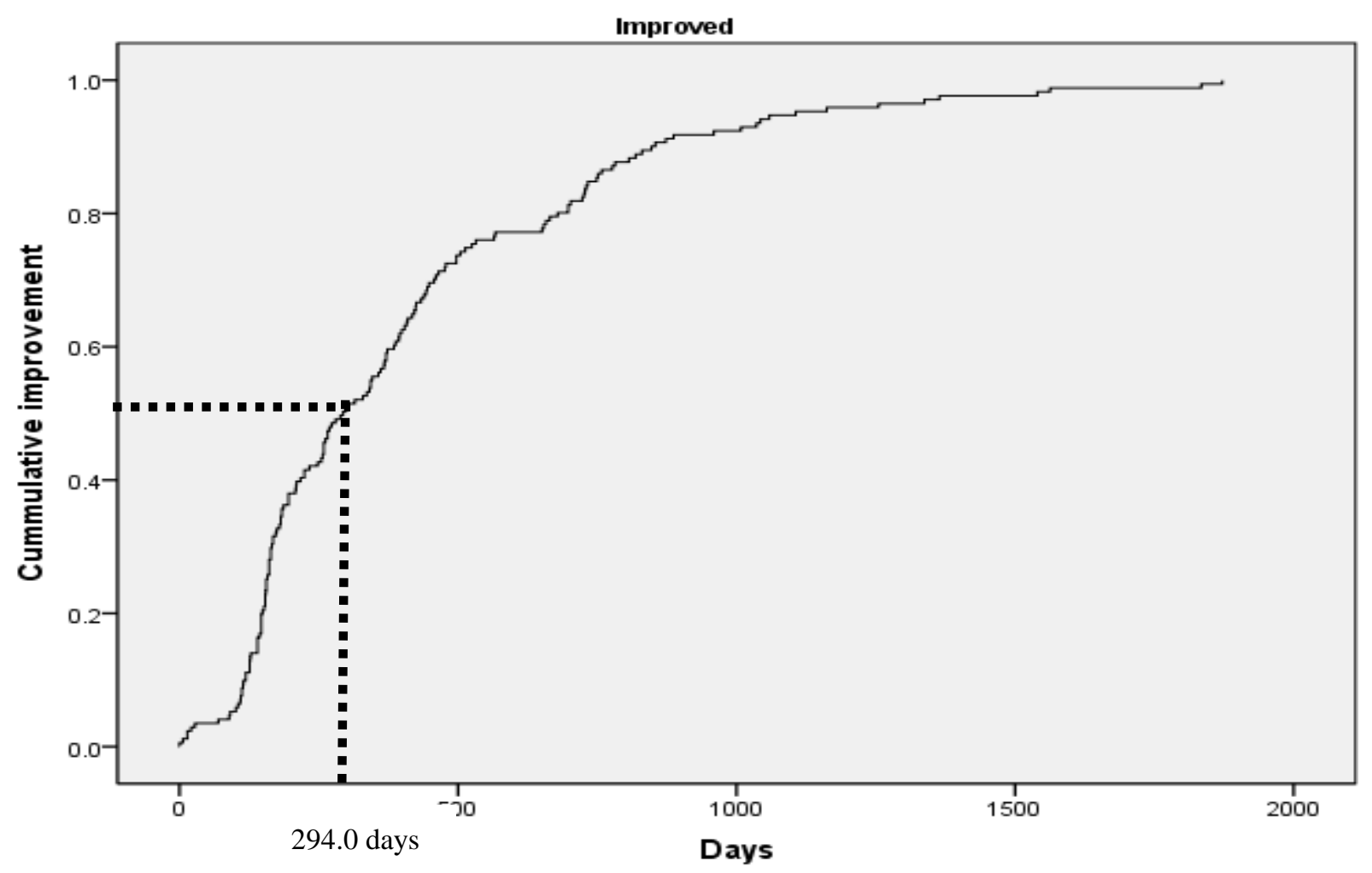

Fig. 3 Survival analysis on treatment of anti-epileptic drugs by Kaplan-Meier. $(n=172)$

In our previous study [4], we found that the incidence of EEG abnormalities decreased by $\geq 90 \%$ in the absence of cephalic ringing. A statistical analysis of EEG abnormalities and trigger factors such as sudden deafness and cephalic ringing showed that the incidence of EEG abnormalities decreased by approximately $70 \%$ in the absence of sudden deafness, and by approximately 50\% in the absence of cephalic ringing. This finding suggests that a considerable number of patients diagnosed with spontaneous sudden deafness, and those who are troubled by hearing difficulties or white noise, may have EEG abnormalities due to CHS.

In light of the results of previous studies, below we propose several new diagnostic criteria for CHS. 


\subsection{Proposed Diagnostic Criteria for CHS (Table 2)}

CHS is a general term for the multiple symptoms, including dizziness and cephalic ringing, which may arise over time when migraine and other forms of chronic headache are not treated appropriately.

The diagnostic criteria for migraine used in this study were the revised international categories for migraine, and we used the ICHD-II for diagnosis. We also considered developing separate criteria for cluster headache, which, like migraine, exhibits the height of cephalic hypersensitivity only in abnormal EEGs. However, the intense pain of cluster headache can cause psychological effects, occurs at intervals of several years, has a tendency to be long and drawn-out, and has an indeterminate end. Therefore, in the present study, we included only a history of migraine and other forms of chronic headache. However, the possibility that patients with tension headache might also be included could not be ruled out, and caution is required on this point. It should also be noted that ordinary migraines also appeared while patients were being treated for CHS. Patients may not have initially noticed the migraines because of the extremely severe cephalic ringing and dizziness; however, as these symptoms were alleviated during the course of treatment, they may have gradually started to recognize the normal migraine symptoms. It is possible that the diagnosis reverts from CHS to migraine at this point.

According to the ICHD, the third edition (beta version; ICHD-III $\beta$ ) [9] published by the Headache Classification Committee of the International Headache Society, "vestibular migraine," which has similar characteristics to CHS, has been newly categorized with its own set of diagnostic criteria. This edition states that patients with migraine who are troubled by moderate to severe vestibular symptoms such as vertigo/dizziness should be diagnosed with "vestibular migraine." However, for now, patients with these characteristics are aligned with our proposed diagnostic criteria (Table 2), which go beyond those of the ICHD by including EEGs as part of the diagnostic criteria.

\subsection{Basic Pathology of CHS (Fig. 4)}

Migraine pain is felt as pain in the prefrontal area of the cerebral cortex when excitatory information via the trigeminal nucleus is transmitted via the thalamus. The cranial and facial allodynia that accompanies the severe migraine mentioned above represents the sensitization of the thalamus and its increased susceptibility to excitation because of the increased excitability of the brain. This increased excitability is caused by a failure to undergo appropriate treatment with triptan medication to stabilize migraine attacks, or by enduring or overlooking the pain of migraine

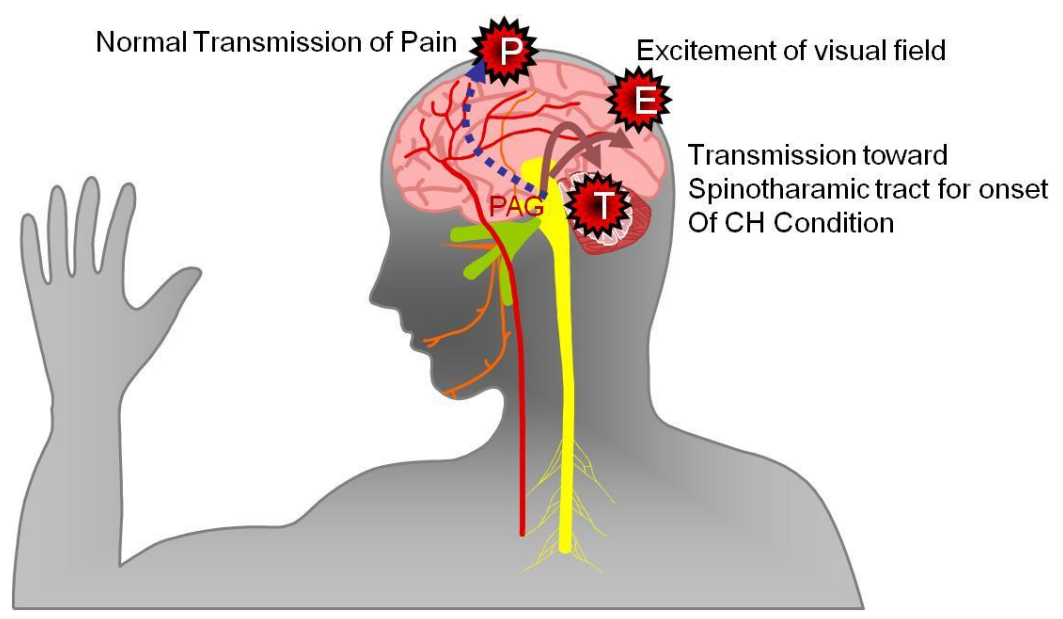

Fig. 4 Mechanism of onset of cephalic hypersensitivity condition.

PAG: periaqueductal grey; $\mathrm{CH}$ : cephalic hypersentivity. 
Table 2 The amended definition of "cephalic hypersensitivity symptoms".

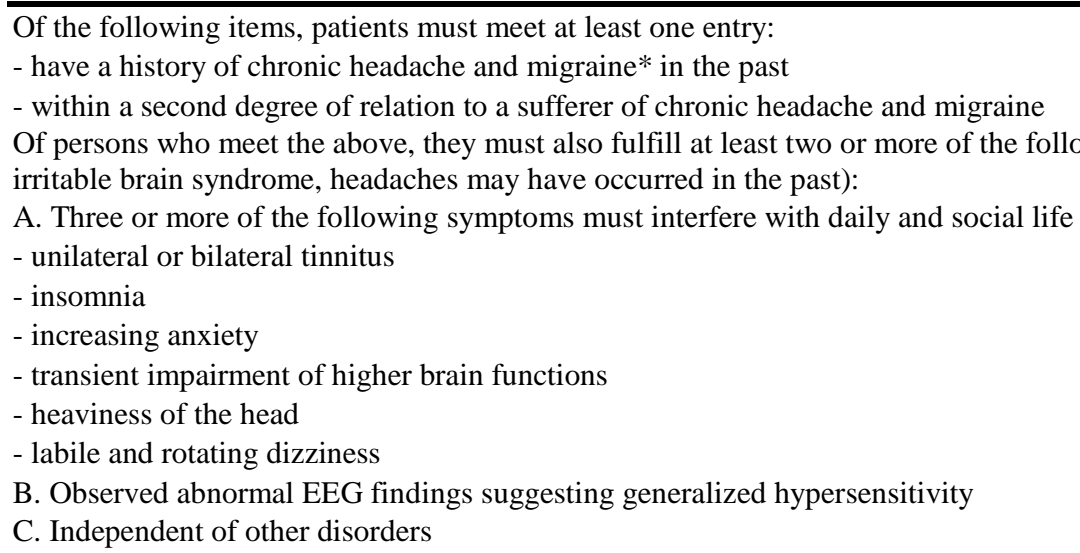

because of the suppression of this excitatory information. This excitability also declines over time, and when it no longer reaches the cerebral cortex, the pain subsides. The basic pathology of migraine according to the trigeminovascular theory is that the trigeminal nerve endings that surround the major cerebral blood vessels also react to the neuroinflammatory proteins that appear in their surroundings during migraines. This causes the expansion and edema of the walls of cerebral blood vessels, which stimulate the trigeminal nerve endings and transmit migraine information to the trigeminal ganglion. However, this vascular expansion is also believed to decrease gradually with age-related arteriosclerosis. One side of the hypothalamus exchanges information with the contralateral cerebellar hemisphere via the thalamocerebellar tract, and this sensitized excitable information may not reach the cerebral cortex, but may instead propagate to the cerebellar hemisphere via the thalamocerebellar tract, appearing as the dizziness associated with CHS. Obstinate cephalic ringing may occur when the auditory area, located in the parietal lobe, is sensitized, or by the weakening of the damping system in the limbic cortex.

\subsection{Treatment of $\mathrm{CHS}$}

As previously described, migraine is the underlying disease for most patients who develop CHS, and anti-epileptics, which have a migraine-preventing effect, are frequently the first-choice drugs for weakening the hyperexcitability of the sensitized brain. Sodium valproate and clonazepam were officially approved in Japan for preventing migraine only recently, and in the United States, topiramate, which can effectively prevent migraine, is also available. If these anti-epileptics are insufficiently effective, the tricyclic antidepressant amitriptyline hydrochloride, which improves the instability of 5-HT in cerebral vessels occurring during migraine and raises the pain threshold, or selective serotonin reuptake inhibitors such as paroxetine hydrate, may also be used successfully in combination. When these medications are prescribed, the patient's symptoms and EEGs should be monitored for changes, and the doses of these drugs should be gradually lowered as EEG findings improve. If the patient starts to recognize normal migraine pain during treatment, the possibility of using triptans, which improve brain excitability, should be considered after confirming the cardiovascular and cerebrovascular stability of the patient's condition. Further, CSD (cortical spreading depression) of Lea is the likely underlying mechanism on development of migraine attack, which can also be alleviated by anti-epileptics as a prophylactic medication. 
The results of our study suggest that erroneous migraine treatment methods from childhood may exacerbate hypersensitivity of the brain and cause the development of dizziness, tinnitus, or cephalic ringing, and show that anti-epileptics are effective for treating this condition.

\section{Conclusion}

Our study suggested that mistaken methods of treatment of migraine from childhood may exacerbate hypersensitivity of the brain and cause the development of dizziness, tinnitus, or cephalic ringing, and that anti-epileptics are effective for treating this condition. Latent varicella zoster virus in the trigeminal ganglion may also be one exacerbating factor that leads to migraine attacks becoming more serious, but further investigation is required.

\section{Acknowledgments}

The authors thank the medical staff at Shiodome Central Clinic for aiding in data collection.

\section{References}

[1] Esposito, M., Marotta, R., Gallai, B., Parisi, L., Patriciello, G., Lavano, S. M., Mazzotta, G., Roccella, M., and Carotenuto, M. 2013. "Temperamental Characteristics in Childhood Migraine without Aura: A Multicenter Study." Neuropsychiatr Dis. Treat. 9: 1187-92.
[2] Ho, T. W., Edvinsson, L., and Goadsby, P. J. 2010. "CGRP and Its Receptors Provide New Insights into Migraine Pathophysiology.” Nat. Rev. Neurol. 6: 573-82.

[3] Shimizu, T., Hirata, K., Manaka, S., and Arakawa, I. 2012. "Physical Disorders and Psychotropics: Treatment of Psychiatric Disturbances Associated with Physical Disorders: Cephalic Hypersensitivity Syndrome." Nihon. Rinsho. 70: 145-50. (in Japanese)

[4] Shimizu, T., Hirata, K., and Arakawa, I. 2010. "Headache Diagnosis by Primary Care Physicians." Iji Shinpo 4251: 40-4.

[5] Ueyama, T., Donishi, T., Ukai, S., Ikeda, Y., Hotomi, M., Yamanaka, N., Shinosaki, K., Terada, M., and Kaneoke, Y. 2013. "Brain Regions Responsible for Tinnitus Distress and Loudness: A Resting State FMRI Study." PLoS One 8: e67778.

[6] Shimizu, T., Hirata, K., Manaka, S., and Arakawa, I. 2013. "Treatment and Social Impact of Cephalic Hypersensitivity Syndrome Relating to Migraine.' Cephalalgia 33: 69-70.

[7] Han, S. S., Nam, E. C., Won, J. Y., Lee, K. U., Chun, W., Choi, H. K., and Levine, R. A. 2012. "Clonazepam Quiets Tinnitus: A Randomised Crossover Study with Ginkgo biloba”. J. Neurol. Neurosurg. Psychiatry 83: 821-7.

[8] International Headache Society (IHS) Classification ICHD-II. 2005. "Diagnostic Criteria for Migraine." Classification (2nd edition-1st revision) Guideline. $\begin{array}{lll}\text { Accessed November } & 7, & \end{array}$ http://www.ihs-headache.org/ichd-guidelines.

[9] International Headache Society (IHS) Classification ICHD-III $\beta$. 2013. "Diagnostic Criteria for Migraine." Classification (3rd edition) Guideline. Accessed November 7, 2013. http://www.ihs-headache.org/ichd-guidelines. 